\title{
Adesão às medidas de biossegurança da enfermagem na unidade de terapia intensiva: revisão sistemática
}

RESUMO | Objetivo: Evidenciar os fatores determinantes para adesão das medidas de biossegurança pela equipe de Enfermagem na Unidade de Terapia Intensiva. Método: Estudo de revisão sistemática, que usou o instrumento Preferred Reporting Items for Systematic Reviews and Meta-Analyses (PRISMA). A busca foi realizada a partir de artigos publicados no período de 2004 a 2019 , por dificuldade de encontrar publicações em menor período acerca da temática deste estudo. Resultados: O conhecimento e as ações de promoção e prevenção, como a lavagem de mãos e o uso de Equipamento de Proteção Individual, além de recursos físicos, são fatores determinantes para adesão às medidas de biossegurança na UTI. Conclusão: As medidas de biossegurança garantem a segurança do paciente, e, por isso, é importante que os profissionais sejam capacitados regularmente para prevenir dificuldades e falhas de adesão com as medidas de biossegurança o que pode colocar em risco o paciente e o profissional da enfermagem.

Palavras-chaves: Enfermagem; Contenção de Riscos Biológicos; Unidades de Terapia Intensiva.

ABSTRACT | Objective: To highlight the determining factors for adherence to biosafety measures by the Nursing team in the Intensive Care Unit. Method: Systematic review study, using the Preferred Reporting Items for Systematic Reviews and MetaAnalyzes (PRISMA) instrument. The search was carried out from articles published from 2004 to 2019 , due to the difficulty of finding publications in a shorter period on the theme of this study. Results: Knowledge and promotion and prevention actions, such as hand washing and the use of Personal Protective Equipment, in addition to physical resources, are determining factors for adherence to biosafety measures in the ICU. Conclusion: Biosafety measures guarantee patient safety, and, therefore, it is important that professionals are trained regularly to prevent difficulties and failures in adherence to biosafety measures, which can put the patient and the nursing professional at risk.

Keywords: Nursing; Containment of Biohazards; Intensive Care Units.

RESUMEN | Objetivo: Resaltar los determinantes de la adherencia a las medidas de bioseguridad por parte del equipo de Enfermería de la Unidad de Cuidados Intensivos. Método: Estudio de revisión sistemática, utilizando el instrumento Preferred Reporting Items for Systematic Reviews and Meta-Analyses (PRISMA). La búsqueda se realizó a partir de artículos publicados de 2004 a 2019, debido a la dificultad de encontrar publicaciones en un período más corto sobre la temática de este estudio. Resultados: El conocimiento y las acciones de promoción y prevención, como el lavado de manos y el uso de Equipos de Protección Personal, además de los recursos físicos, son factores determinantes para el cumplimiento de las medidas de bioseguridad en la UCI. Conclusión: Las medidas de bioseguridad garantizan la seguridad del paciente, por lo que es importante que los profesionales se capaciten periódicamente para prevenir dificultades y fallas en la adherencia a las medidas de bioseguridad, que pueden poner en riesgo al paciente y al profesional de enfermería.

Palavras claves: Enfermería; Contención de Riesgos Biológicos; Unidades de Cuidados Intensivos.

\section{Karina Polyana Costa}

Graduanda de Enfermagem, Universidade do Estado de Minas Gerais, Brasil, unidade acadêmica de Divinópolis, Minas Gerais, Brasil. ORCID: 0000-0001-9796-2780

\section{Alexandre Coutinho de Melo}

Graduando de Enfermagem, Universidade do Estado de Minas Gerais, Brasil, unidade acadêmica de Divinópolis, Minas Gerais. ORCID: 0000-0001-5767-9796

\section{Bruno Barbosa Silva}

Graduando de Enfermagem, Universidade do Estado de Minas Gerais, Brasil, unidade acadêmica de Divinópolis, Minas Gerais, Brasil. ORCID: 0000-0002-3799-5131

\section{Isabele Adriana Costa}

Graduanda de Enfermagem, Universidade do Estado de Minas Gerais, Brasil, unidade acadêmica de Divinópolis, Minas Gerais. Brasil. ORCID: 0000-0002-6844-1553

\section{Leonardo Gomes de Freitas}

Enfermeiro docente do Curso de Enfermagem, Universidade do Estado de Minas Gerais, unidade acadêmica de Divinópolis, Minas Gerais, Brasil. ORCID: 0000-0003-2008-7054

\section{Regina Consolação dos Santos}

Enfermeira docente do Curso de Enfermagem, Universidade do Estado de Minas Gerais, unidade acadêmica de Divinópolis, Minas Gerais, Brasil.

ORCID: 0000-0002-7393-3210

\section{Silmara Nunes Andrade}

Enfermeira docente do Curso de Enfermagem, Universidade do Estado de Minas Gerais, unidade acadêmica de Divinópolis, Minas Gerais, Brasil.

ORCID: 0000-0002-1975-0827

Recebido em: 29/07/2020

Aprovado em: 14/08/2020

\section{Débora Aparecida Silva Souza}

Enfermeira docente do Curso de Enfermagem, Universidade do Estado de Minas Gerais, unidade acadêmica de Divinópolis, Minas Gerais, Brasil. ORCID: 0000-0002-8937-584X

INTRODUÇÃO

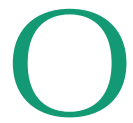
exercício da Enfermagem é marcado pela exposição de riscos físicos, químicos e biológicos, que colocam os profissionais no pilar sobre a importância das medidas de biossegurança. Tais medidas caracterizam-se por ações de precaução-padrão que estão diretamente ligadas à prevenção, ao controle, à redução ou à eliminação dos riscos ocupacionais a que os profissionais estão expostos. ${ }^{(1)}$

O papel do enfermeiro perante a implementação da NR 32 visa influenciar e 
capacitar seus colabores a prestarem uma assistência à saúde de qualidade e se de promover a promoção, recuperação, assistência, pesquisa e ensino em saúde em qualquer nível de complexidade.(2)

No contexto da Unidade de Terapia Intensiva (UTI), as medidas de biossegurança são indispensáveis à vida e à saúde dos pacientes, independentemente do seu diagnóstico, além da proteção e garantia da integridade física do profissional neste ambiente crítico. ${ }^{(3)}$

$\mathrm{Na}$ Enfermagem, a higienização das mãos com água e sabão, a fricção alcoólica e o uso de máscara de proteção respiratória, óculos, luvas de procedimento e estéril, jaleco, avental, capote e gorro são medidas de biossegurança rotineiramente realizadas no exercício da profissão. ${ }^{(4-5)}$ Embora relativamente simples e de custo baixo, tais medidas ainda representam um desafio na adesão entre os profissionais, necessitando de aprofundamento de estudos que justifiquem os fatores determinantes por estas falhas. ${ }^{(2)}$

Estudo realizado em um hospital-escola evidenciou que infecções hospitalares em unidades críticas, como UTI, apresentam taxas de infecção hospitalar significativas, quando comparadas a outros setores do hospital. ${ }^{5}$ Resultados como este não são amplamente divulgados, embora haja uma estimativa de que infecções hospitalares, e em torno de 17 milhões de pessoas por ano são contaminadas no mundo. ${ }^{(6)}$

Este achado ilustra um agravo à saúde que coloca em risco a segurança do paciente e que pode ser desencadeado pela não adesão de uma medida de biossegurança, principalmente em ambientes críticos como a UTI, onde são realizados procedimentos de maior complexidade. ${ }^{(5)}$

O Ministério do Trabalho instituiu, no Brasil, a Norma Regulamentadora $n^{\circ} 32$ (NR 32) que visa estabelecer diretrizes e implantações para os estabelecimentos de saúde em relação à proteção e segurança dos trabalhadores e aos profissionais de saúde, e pela Norma Regulamentadora $n^{\circ} 06$ (NR 06) que visa o uso de Equipamento Individual de Proteção (EPI), destinada à proteção da integridade dos trabalhadores e de prevenir doenças ocupacionais. Estas implantações englobam as medidas preventivas com um custo baixo e eficaz, porém não são muito utilizadas pelos profissionais. Um exemplo é a dificuldade do uso de Equipamento de Proteção Individual (EPI). ${ }^{(2)}$

De acordo com Pereira et $\mathrm{al}^{(4)}$, os profissionais de Enfermagem possuem conhecimento sobre as medidas de biossegurança, mas isto não implica em uma adesão satisfatória, pois pode causar disseminação de microrganismos, o que gera sofrimento ao paciente por dificultar o seu tratamento na UTI, além de acarretar ônus ao sistema de saúde. ${ }^{(7)}$

Por esta razão, a atenção às questões de biossegurança necessita de destaque na literatura por retratar riscos muitas vezes irreparáveis mediante situações que poderiam ser prevenidas. Dessa forma, este estudo teve como objetivo conhecer os fatores determinantes para adesão às medidas de biossegurança pela equipe de Enfermagem na UTI.

\section{MÉTODO}

Trata-se de uma revisão sistemática, que se classifica como um estudo secundário, visando a trabalhar a partir de outras pesquisas de origem primária. É importante destacar que este estudo foi realizado de forma clara, utilizando métodos sistemáticos e explícitos na revisão. ${ }^{(4-8-9)}$ Contudo, é necessária uma sistematização em todo o seu processo. Desta forma, o presente estudo utilizou o instrumento Preferred Reporting Items for Systematic Reviews and Meta-Analyses (PRISMA), garantindo a veracidade na pesquisa. ${ }^{(4-8-9)}$

Inicialmente, a busca partiu de um critério de autenticidade, em que se estabeleceu a estratégia "PICOS": "P" (pacients): profissionais de Enfermagem de ambos os sexos que atuam em UTI; "I" (intervention): prática que se faz necessária no intuito de reduzir os riscos de contaminação e acidentes no trabalho baseada na NR 32(6): " $C$ " (control): ações que vão contra as normas regulamentadoras de biossegurança; "O" (outcomes): eventos adversos; $\mathrm{e}$ "S" (study design): exploratórios ensaios clínicos randomizados e observacionais (transversal, coorte e caso-controle). Diante disto, a pergunta norteadora formada da pesquisa foi a seguinte: "Quais fatores determinam a adesão às práticas de biossegurança na UTI?"(4-8)

Posteriormente, foi realizada a busca nas bases de dados Literatura Latino-Americana e do Caribe em Ciências da Saúde (LILACS), Scientific Electronic Library Online (SciELO), Índice Bibliográfico Español en Ciencias de la Salud (IBECS), Medical Literature Analysis and Retrieval System Online (MEDLINE) e Bases de Dados de Enfermagem (BDENF), por meio dos seguintes descritores disponíveis no Descritores em Ciências da Saúde (DeCS): Enfermagem, Biossegurança e Unidades de Terapia Intensiva.

A segunda etapa partiu da busca de estudos no período dos últimos cinco anos, de 2014 a 2019. Contudo, como foi evidenciada escassez de estudos, a busca foi ampliada com artigos publicados no período de 2004 a 2019. Foram adotados, como critérios de inclusão, artigos em português, interesse do que os dados revelariam no Brasil, texto disponível na íntegra e artigos originais qualitativos e quantitativos que justificassem os motivos de adesão às medidas de biossegurança na equipe de Enfermagem na UTI.

Foram excluídos os artigos não disponíveis on-line, com publicações que não remetessem ao período de busca e temática voltadas para a percepção e o conhecimento dos profissionais de Enfermagem nas práticas de segurança biológica na UTI.

A coleta de dados ocorreu mediante a seleção dos artigos que atenderam ao objetivo deste estudo, a partir da leitura na íntegra de cada artigo, para verificar se estes responderiam à pergunta desta revisão sistemática da literatura. Em seguida, foi construída uma planilha com autores, ano da publicação, delineamento meto- 
dológico, fatores de adesão e resultados (Tabela 1). A construção deste banco de dados teve como finalidade oferecer suporte para a quarta e quinta etapas da revisão sistemática: análise crítica e discussão dos dados encontrados.

Outro ponto a ser considerado é como ocorreu a extração dos dados - que teve três revisores (A. C. M., K. P. e B. E. F) -, na qual foram realizadas a remoção dos artigos publicados e a avaliação dos artigos pelo título e pelos resumos. Posteriormente, os artigos selecionados foram lidos na íntegra, com discussão crítica e parecer de cada revisor. Por fim, artigos que tiveram alguma discordância dos revisores, no que se refere à inclusão ou exclusão, tiveram de ser relidos para nova avaliação e consenso.

Em relação às variáveis, foram extraídas dos artigos selecionados para análise (Figura 1): (1) autor, ano de publicação e país de publicação; (2) fatores de adesão em relação à biossegurança; (3) medidas de não adesão; e (4) principais resultados.

Por se tratar de uma revisão sistemática da literatura, este estudo não apresentou a necessidade de apreciação e aprovação pelo Comitê de Ética em Pesquisa, visto que os dados não precisam de sigilo ético.

\section{RESULTADOS}

Ao todo, foram encontrados nove artigos na busca de dados descritivos. Em seguida, houve a remoção dos duplicados, que foram excluídos pelo título ou pelo resumo, restando cinco artigos que foram avaliados em seus títulos e resumos. Utilizando-se os critérios de elegibilidade, apenas três artigos foram selecionados na íntegra (Figura 1), entre os quais um sobre coorte-transversal quantitativo e dois de pesquisa exploratória com abordagem qualitativa dos dados (Tabela 1).

Dessa forma, o primeiro estudo de Llapa-Rodriguez et al $^{(2)}$ teve como objetivo avaliar as recomendações de biossegurança junto aos profissionais de Enfermagem na UTI. Utilizando-se de uma abordagem quantitativa, descritiva e de corte transversal, foi possível elencar que os profissionais de Enfermagem têm o conhecimento sobre biossegurança, porém isso não garante o cumprimento das normas técnicas definidas para os profissionais devido à indisponibilidade na unidade, gerando, assim, o desmazelo com a utilização dos EPIs. O estudo evidenciou o aprendizado sobre os procedi-

\section{Figura 1 - Seleção dos artigos por estratégia de busca nas bases de dados.}

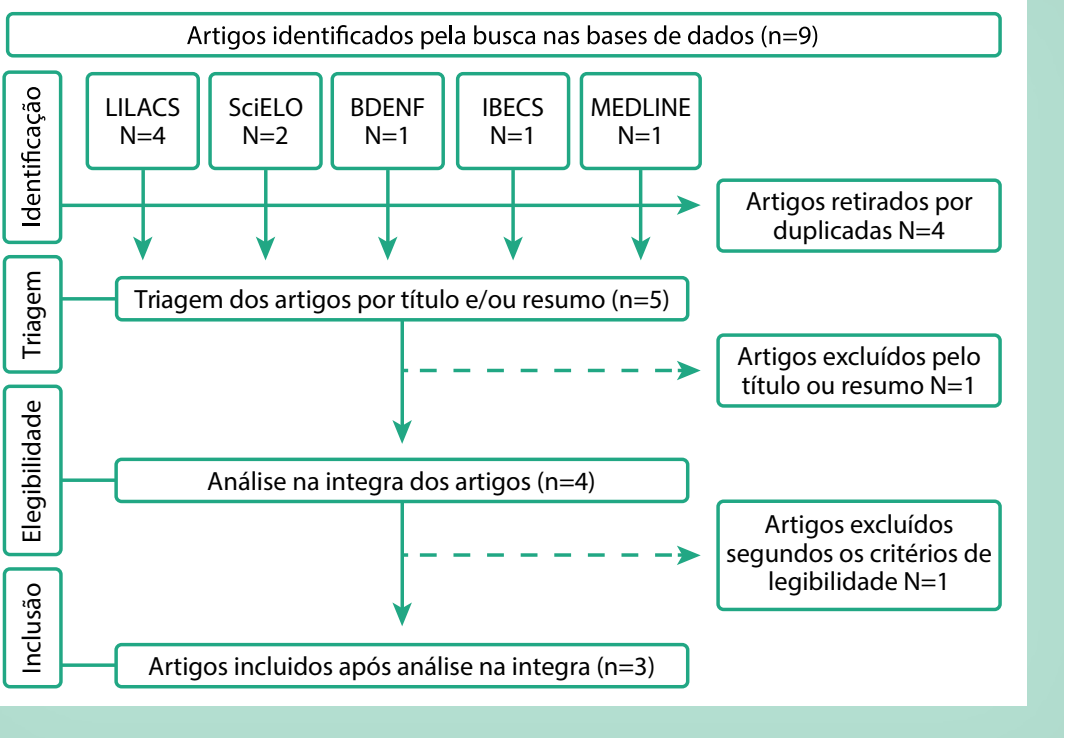

mentos de biossegurança e as ações de promoção e prevenção sobre segurança do trabalho, como, por exemplo, o conhecimento sobre lavagem de mãos e uso de EPI, favorecendo a adesão às medidas de biossegurança na UTI.

Já em Correa e Donato ${ }^{(10)}$, estudo tipo exploratório, descritivo e com uma abordagem qualitativa, buscou-se descrever as ações realizadas pela equipe de Enfermagem durante o processo de assistência na UTI para que fosse possível identificar a efetividade das medidas de biossegurança durante a assistência. Além disso, foram analisadas as possibilidades de implementação na equipe de medidas de biossegurança durante a assistência. Este estudo possibilitou a reflexão da necessidade de se promover uma educação permanente para os colaboradores, além de ações de promoção e prevenção em segurança do trabalho e de implementação das "boas práticas", possibilitando alçar um ambiente de trabalho com menos risco de acidentes ocupacionais. Para os autores, investir em recursos físicos nas UTIs e ampliar os conhecimentos dos profissionais, como, por exemplo, sobre a importância da higienização das mãos com água e sabão e do conhecimento a respeito do uso de $\mathrm{EPI}$, repercutem na adesão destas medidas.

Assim também o estudo de Brand e Fontana(11) objetivou investigar saberes e práticas da equipe de Enfermagem sobre biossegurança na UTI, bem como identificar situações de risco biológico a que o trabalhador está exposto, e a adesão à NR 32. Utilizando uma abordagem qualitativa, o estudo descritivo possibilitou refletir sobre as normas e os responsáveis por promover a biossegurança dos setores, mesmo que ainda haja uma aderência completa das instituições. Nesse sentido, os serviços prestados pelos órgãos responsáveis são mais utilizados de forma fiscalizatória do que educativa e/ou emancipatória, oferecendo educação continuada para que haja formas assertivas de adesão às medidas de biossegurança. 


\section{DISCUSSÃO}

Nos estudos elencados para esta revisão sistemática, foi possível evidenciar que o conhecimento sobre os procedimentos de biossegurança, as ações de promoção e prevenção sobre segurança do trabalho, os recursos físicos adequados das UTIs, a capacitação dos profissionais para ampliar o conhecimento técnico e científico e, ainda, as ações educativas desenvolvidas pelos setores responsáveis são fatores determinantes para a adesão às medidas de biossegurança na UTI.

Os dados disponíveis na literatura são ainda escassos e limitados no sentido de permitir que se identifiquem as melhores medidas para que se estabeleça a forma mais adequada de se garantir as recomendações da NR 32, sendo uma norma regulamentadora que estabelece as diretrizes básicas para a implementação de medidas de proteção à segurança e a saúde dos trabalhadores em serviços de saúde. E, embora seja um tema discutido há algumas décadas, ainda é evidente a falta de adesão nas equipes de saúde que consideram, também, o excesso de trabalho, o estresse, a falta de equipamento e até mesmo a estrutura física do ambiente de trabalho.

No que concerne à adesão das medidas de biossegurança nas equipes de saúde, enaltecemos o uso da ferramenta da educação continuada, que é um artifício criado por Freire ${ }^{(12)}$. Este recurso faz com que os profissionais elenquem fatores de sua vida profissional e pessoal na prática do seu cuidado, criando mais associações do seu trabalho com seu conhecimento e estabelecendo, de uma maneira crítica, a importância de se realizar as medidas de biossegurança. ${ }^{(13)}$

Estabelecendo uma conexão com a educação continuada, é importante um olhar crítico sobre a educação permanente, que é ressaltada nos estudos de Silva et $\mathrm{al}^{(14)}$, pois esta é apresentada como uma demanda elencada nos artigos estudados. A educação continuada permite adotar medidas para desfrutar as oportunidades de aprendizagem dos profissionais e já na educação permite nortear e promover processos de problematização do processo de trabalho. ${ }^{(15)}$ Há uma falta de conhecimento até mesmo durante a graduação sobre a terminologia biossegurança, além do que, a depender da instituição, podem ocorrer pequenas mudanças que variam conforme a filosofia do trabalho.

\section{C6}

Diante disto, pensar no outro é refletir que o ser humano necessita de cuidados integrais e dinâmicos, indo além do contato físico. Há, também, a necessidade de se ter um espaço seguro e pessoas sensibilizadas para cuidar de uma maneira que possibilite diminuir ao máximo os danos.

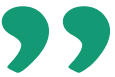

Desta maneira, é necessário aprofundar a temática e pensar na aplicabilidade destas medidas nas instituições como uma forma educativa, atingindo todos os profissionais, pois as medidas educativas citadas nos artigos são as mais eficientes.
No entanto, planejar formas de se alcançar estes objetivos vai além das necessidades de investimento em infraestrutura. A estrutura física abordada em relação à UTI é um local de alta complexidade, que admite pacientes com um potencial grave. Mesmo assim, medidas de biossegurança nestes ambientes ainda são falhas, como, por exemplo, a falta de dispersores de álcool em gel e de pias para a higienização das mãos dos profissionais, dificultando o cumprimento da NR 32.(2)

De acordo Ministério da Saúde é estabelecido pela ANVISA a RDC 50 da Resolução $n^{\circ} 7$ de fevereiro de 2010, que tem por objetivo planejar, programar, elaborar e avaliar os projetos e estruturas físicas da UTI. ${ }^{(16)}$

Diante disto, pensar no outro é refletir que o ser humano necessita de cuidados integrais e dinâmicos, indo além do contato físico. Há, também, a necessidade de se ter um espaço seguro e pessoas sensibilizadas para cuidar de uma maneira que possibilite diminuir ao máximo os danos.

É importante destacar, dentro da temática de adesão às medidas de biossegurança, a segurança do paciente. Este movimento de empatia com outro a ser cuidado ressalta também a importância da segurança do paciente, que está notoriamente atrelada à NR de biossegurança, uma vez que, realizando-a mutuamente, consegue-se estabelecer formas efetivas de cuidado com a segurança, diminuindo acidentes tanto com os pacientes como com a equipe de saúde. ${ }^{(17)}$ Entretanto, um aspecto citado nos estudos é a não utilização de equipamentos individuais, fator crucial para cumprir com as medidas de biossegurança. O uso dos equipamentos é um dos mais ressaltados pelos participantes nos artigos elencados, pois a utilização dos EPIs permite barreiras físicas, evitando acidentes e infecções transversais. ${ }^{(17)}$ Atrelado aos equipamentos ressaltou-se a importância da lavagem de mãos e da utilização do jaleco - estes dois "itens" devem obedecer à NR 32 para que sejam evitadas contaminações. ${ }^{(11)}$ 
Estudo realizado por Vasconcelos et al(18) mostra que a lavagem de mãos é a forma mais eficaz e barata para evitar a contaminação transversal. Além disso, evita infecções que podem afetar o próprio profissional. Os autores destacam que a não lavagem de mão, como ressaltado nos estudos, muitas vezes é justificada pelo tempo e pela sobrecarga de trabalho. Encontrar meios de se valorizar essas ações é uma forma de visibilizar a utilização destas ferramentas. Pesquisa realizada por Brand e Fontana ${ }^{(11)}$ mostrou que, quando há diálogo e dinâmica entre a equipe, há, também, uma melhora em relação ao fato de que é necessário seguir as orientações de biossegurança.

Este estudo considera como limitação a dificuldade de publicações disponíveis que evidenciam investigações que aprofundem a visão dos profissionais de En- fermagem sobre os fatores que facilitam e contribuem para a adesão às medidas de biossegurança na UTI. Embora tenha seguido um rigor metodológico, o estudo não permite associações, por se tratar de uma pesquisa qualitativa.

\section{CONCLUSÃO}

A UTI é um ambiente crítico e que demanda atenção, devido à complexidade clínica dos pacientes, além da diversidade de procedimentos invasivos realizados por toda a equipe de Enfermagem. Apesar da escassez de publicações realizadas com a temática, fica claro que os principais fatores determinantes para a adesão são ás medidas de biossegurança na Unidade de Terapia Intensiva (UTI), como o conhecimento dos profissionais de enfermagem e as ações de prevenções e promoções como, por exemplo, a higienização das mãos e o uso do Equipamento Individual de Proteção (EPI), e além da melhora das estruturas e recursos físicos, ou seja, possibilitar um ambiente de trabalho com menos riscos de acidentes ocupacionais e de se promover educação continuada para que haja formas assertivas de adesão ás medidas de biossegurança, possibilitando medidas de biossegurança e implementações pela equipe de enfermagem e de se promover a segurança do paciente. Estes fatores são cruciais para que se realize a segurança do paciente de uma forma eficiente, uma vez que esta permite desenvolver os aspectos de segurança do trabaIhador e, também, a forma de se realizar o cuidado. Portanto, sugere-se a realização de mais pesquisas na área, ressaltando a importância da perspectiva do profissional de Enfermagem.

\section{Referências}

1. Cvejanov-Kezunovic L, Mustajbegovi囚 J, Milosevic M, Civljak R. Occupational exposure to blood among hospital workers in Montenegro. Arh Hig Rada Toksikol. 2014; 65(3):273-80. doi: 10.2478/10004-1254-65-20142493.

2. Llapa-Rodriguez EF, Silva GG, Neto DL, Campos MPA, Mattos MCT, Otero LM. Medidas para adesão às recomendações de biossegurança pela equipe de Enfermagem. Enferm Glob 2018; 17(49):36-67. doi: https://doi. org/10.6018/eglobal.17.1.276931.

3. Almeida NR, Carvalho BJDF, Neta ABN, Queiróz KSP. Perfil epidemiológico das infecções relacionadas à assistência à saúde em Unidades de Terapia Intensiva. Cad ESP (Ceará). 2015; 9(1):42-51.

4. Pereira FMV, Malaguti-Toffano SE, Silva AM da, Canini SRMS, Gir E. Adesão às precauções-padrão por profissionais de Enfermagem que atuam em terapia intensiva em um hospital universitário. Rev Esc Enferm USP [Internet]. 2013; 47(3):686-693. doi: http://dx.doi.or g/10.1590/50080623420130000300023

5. Souza ES, Belei RA, Carrilho CMD de M et al. Mortalidade e riscos associados a infecção relacionada à assistência à saúde. Texto Contexto - Enferm. [Internet]. 2015; 24(1):220-228. doi: http://dx.doi.org/10.1590/010407072015002940013

6. Abegg PTGM, Silva LL. Controle de infecção hospitalar em Unidade de Terapia Intensiva: estudo retrospectivo. Semina Cienc Biol Saude. 2011; 32(1):47-58. doi: 10.5433/1679-0367.2011v32n1p47.

7. Santos TCR dos, Roseira CE, Piai-Morais TH, Figueiredo RM de. Hand hygiene in hospital environments: use of conformity indicators. Rev Gaúcha Enferm. [Internet]. 2014; 35(1):70-77. doi: http://dx.doi.org/ 10.1590/19831447.2014.01.40930.

8. Galvão TF, Pereira MG. Systematic reviews of the literature: steps for preparation. Epidemiol Serv Saúde [Internet]. 2014; 23(1):183-184. doi: http:// dx.doi.org/10.5123/S1679-49742014000100018.

9. Moher D, Shamseer L, Clarke M et al. Preferred reporting items for systematic review and meta-analysis protocols (PRISMA-P) 2015 statement. Syst Rev. 2015; 1(4):1-9. doi: 10.1186/2046-4053-4-1.
10. Correa CF, Donato M. Biossegurança em uma unidade de terapia intensiva: a percepção da equipe de enfermagem. Esc Anna Nery [Internet]. 2007; 11(2):197-204. http://dx.doi.org/10.1590/S1414-81452007000200003.

11. Brand $\mathrm{Cl}$, Fontana RT. Biossegurança na perspectiva da equipe de enfermagem de Unidades de Tratamento Intensivo. Rev Bras Enferm [Internet]. 2014; 67(1):78-84. doi: http://dx.doi.org/10.5935/0034-71 67.20140010.

12. Freire P. Pedagogia da autonomia: saberes necessários à prática educativa. 9. ed. Rio de Janeiro: Paz e Terra; 1998.

13. Silva GM da, Seiffert OMLB. Educação continuada em enfermagem: uma proposta metodológica. Rev Bras Enferm [Internet]. 2009; 62 (3):362-366. doi: http://dx.doi.org/10.1590/\$0034-716720090003000 05.

14. Silva M F, Conceição F A, Leite, M M J. Educação continuada: um levantamento de necessidades da equipe de Enfermagem. Arq Bras Ciên Saúde. 2009; 34(1):15-21.

15. Paschoal A S, Mantovani $M$ de F, Méier $M$ J. Percepção da educação permanente, continuada e em serviço para enfermeiros de um hospital de ensino. Rev Esc Enferm USP [Internet]. 2007; 41(3):478-484. doi: http:// dx.doi.org/10.1590/50080-62342007000300019.

16. Brasil. Ministério da Saúde. Documento de referência para o Programa Nacional de Segurança do Paciente. Brasília: Ministério da Saúde/ Agência Nacional de Vigilância Sanitária. RDC 50. 2002. [acesso 2019 dez. 05]. Disponivel em: http://bvsms.saude.gov.br/bvs/saudelegis/anv isa/2010/ res0007_24_02_2010.html.

17. Brasil. Ministério da Saúde. Documento de referência para o Programa Nacional de Segurança do Paciente. Brasília: Ministério da Saúde/Fundação Oswaldo Cruz/Agência Nacional de Vigilância Sanitária, 2014. [acesso 2019 out. 02]. Disponível em: http://bvsms.saude.gov.br/bvs/publicacoes/documento_referencia_programa_nacional_seguranca.pdf.

18. Vasconcelos R O, Alves D C I, Fernandes L M, Oliveira J L C de. Adesão à higienização das mãos pela equipe de Enfermagem em Unidade de Terapia Intensiva. Enferm Glob 2018; (50):446-461. doi: 10.6018/eglobal.17.2.284131. 